\title{
Simplified coronary flow reserve calculations based on three-dimensional coronary reconstruction and intracoronary pressure data
}

\author{
Benjamin $\mathrm{Csippa}^{1}$, Áron Üveges ${ }^{2,3,4}$, Dániel Gyürki ${ }^{1}$, Csaba Jenei ${ }^{2,4}$, \\ Balázs Tar ${ }^{3,4}$, Balázs Bugarin-Horváth ${ }^{3}$, Gábor Tamás Szabó ${ }^{2,4}$, \\ András Komócsi ${ }^{5}$, György Paál ${ }^{1}$, Zsolt Kőszegi ${ }^{2,3,4}{ }^{4}$ \\ ${ }^{1}$ Department of Hydrodynamic Systems, Budapest University of \\ Technology and Economics, Budapest, Hungary \\ ${ }^{2}$ Division of Cardiology, Faculty of Medicine, University of Debrecen, Hungary \\ ${ }^{3}$ Szabolcs-Szatmár-Bereg County Hospitals and University Teaching Hospital, Nyíregyháza, Hungary \\ ${ }^{4}$ Kálmán Laki Doctoral School of Biomedical and Clinical Sciences, University of Debrecen, Hungary \\ ${ }^{5}$ Heart Institute, Medical School, University of Pécs, Hungary
}

\begin{abstract}
Background: Measurements of fractional flow reserve (FFR) and/or coronary flow reserve (CFR) are widely used for hemodynamic characterization of coronary lesions. The frequent combination of the epicardial and microvascular disease may indicate a need for complex hemodynamic evaluation of coronary lesions. This study aims at validating the calculation of CFR based on a simple hemodynamic model to detailed computational fluid dynamics (CFD) analysis.

Methods: Three-dimensional (3D) morphological data and pressure values from FFR measurements were used to calculate the target vessel. Nine patients with one intermediate stenosis each, measured by pressure wire, were included in this study.

Results: A correlation was found between the determined CFR from simple equations and from a steady flow simulation $\left(r=0.984, p<10^{-5}\right)$. There was a significant correlation between the CFR values calculated by transient and steady flow simulations $\left(r=0.94, p<10^{-3}\right)$.

Conclusions: Feasibility was demonstrated of a simple hemodynamic calculation of CFR based on $3 D$-angiography and intracoronary pressure measurements. A simultaneous determination of both the FFR and CFR values provides the capability to diagnose microvascular dysfunction: the CFR/FFR ratio characterizes the microvascular reserve. (Cardiol J)
\end{abstract}

Key words: coronary flow reserve, fractional flow reserve, microvascular function, three-dimensional reconstruction

\section{Introduction}

Measurements of fractional flow reserve (FFR) and/or coronary flow reserve (CFR) are widely used for hemodynamic characterization of coronary stenosis. An FFR value of 0.80 , cal- culated as the mean distal and proximal coronary pressure in hyperemia, is the threshold below which percutaneous coronary intervention (PCI) and stent implantation are recommended [1-4]. Multiple clinical studies demonstrated that FFR is a consistent metric to support decision-making

Address for correspondence: Zsolt Kőszegi, MD, PhD, Division of Cardiology, Faculty of Medicine, University of Debrecen, Móricz Zs. krt. 22, H-4032 Debrecen, Hungary, tel/fax: +36 52 255928, e-mail: koszegi@med.unideb.hu 
in treatment options during heart catheterization [1-4]. However, despite the highly significant difference between the PCI and the medical therapy groups in the primary endpoint [5], the risk assessment potential of FFR on the natural history can be interpreted to be limited. Therefore, the need for a more complex hemodynamic evaluation of coronary lesions is warranted.

Coronary flow reserve is defined as the ratio of a cycle-averaged coronary flow rate in the stenosed artery segment during maximum vasodilation and the resting condition. A threshold of hemodynamic significance is proposed at coronary lesions with CFR $<2.0$. In contrast to FFR that addresses the hemodynamic consequences of focal stenosis of the conductance vessels, CFR could be the subject of processes that involve the entire coronary artery system. Thus CFR can also be impaired due to microvascular dysfunction originating from atherosclerotic or non-atherosclerotic processes [6-11]. CFR was an independent factor of mortality in patients with suspected coronary artery disease, providing incremental risk stratification over clinical variables and perfusion imaging $[12,13]$.

Despite the correlation between CFR and FFR, a relevant discrepancy between these two parameters may occur, reflecting distinct aspects of the coronary pathophysiology [14]. These differences underline the importance of a comprehensive description of stenosis physiology by both the pressure and flow characteristics [15].

The resistance of the microvascular system is the ratio of the distal coronary pressure divided by the distal coronary flow. During maximal vasodilation, the hyperemic (minimal) resistance of the microcirculation can be characterized by the thermodilution method by calculating the index of microcirculatory resistance (IMR): $\mathbf{I M R}=$ mean transit time $\times$ distal coronary pressure.

While the IMR is influenced significantly by the perfused myocardial mass [7-10], the Doppler flow velocity measurement shows the hyperemic microvascular resistance (HMR) without this limitation but with the laborious technical problems of the Doppler measurement. In cases when both the FFR and the CFR can be determined, the CFR/FFR ratio will provide useful information about microvascular resistance; $<2$ value indicates microvascular dysfunction [16].

The microvasculature has become focused on the pathogenesis of coronavirus disease 2019 (COVID-19)-related cardiovascular involvement. The role of microvascular injury has arisen both in the acute phase and in the post-COVID cardiac syndrome provoked by possible immune-mediated mechanisms [17, 18].

Such a diagnostic approach that can provide information on the microvasculature without having to apply further invasive device testing during a routine FFR measurement may serve as good guidance for follow-up on COVID-19-related microvascular abnormalities [19].

The present study aims at establishing a clinically applicable method for simultaneous CFR determination during a routine FFR measurement. We have developed a simple three-dimensional (3D) angiography- and intracoronary pressure measurement-based hemodynamic calculations which do not require the time-consuming modeling of the flow. In this paper, this simple method was validated by the results of detailed unsteady and steady-state modeling of the flow using a finite volume method that solves the Navier-Stokes and continuity equations.

\section{Methods}

\section{Study population}

Nine patients ( 4 males, 5 females) who underwent invasive pressure wire measurement were selected for this retrospective study from 20 consecutive patients with stable coronary artery disease. Inclusion criteria were single well-defined intermediate stenosis (40-70\% diameter stenosis by visual assessment) in a main branch of the coronary system with a diameter $>2 \mathrm{~mm}$, appropriate angiographic views for $3 \mathrm{D}$ reconstructions, and good quality hyperemic and resting pressure traces with checking for pressure drift by the pullback of the pressure sensor at the end of the procedure. In addition, patients with an acute coronary syndrome left main coronary artery stenosis, ostial stenosis, previous coronary artery bypass graft surgery, or diffuse coronary artery disease were excluded. Segment detection, 3D reconstruction, and pressure measurement of 1 case are presented in Figure 1.

Detection of more than $2 \mathrm{mmHg}$ drift at the end of the measurement was also an exclusion criterion. Among the 20 screened patients, 5 cases did not have appropriate angiograms for 3D coronary artery reconstruction from two angiographic views (with at least $25^{\circ}$ difference), and in 6 cases more than $2 \mathrm{mmHg}$ drift was detected and was a cause for exclusion. The remaining 9 patients were analyzed.

The present study was performed in concordance with the ethical standards laid down in the 1964 Declaration of Helsinki and its later amendments and approved by the Hungarian Office of 


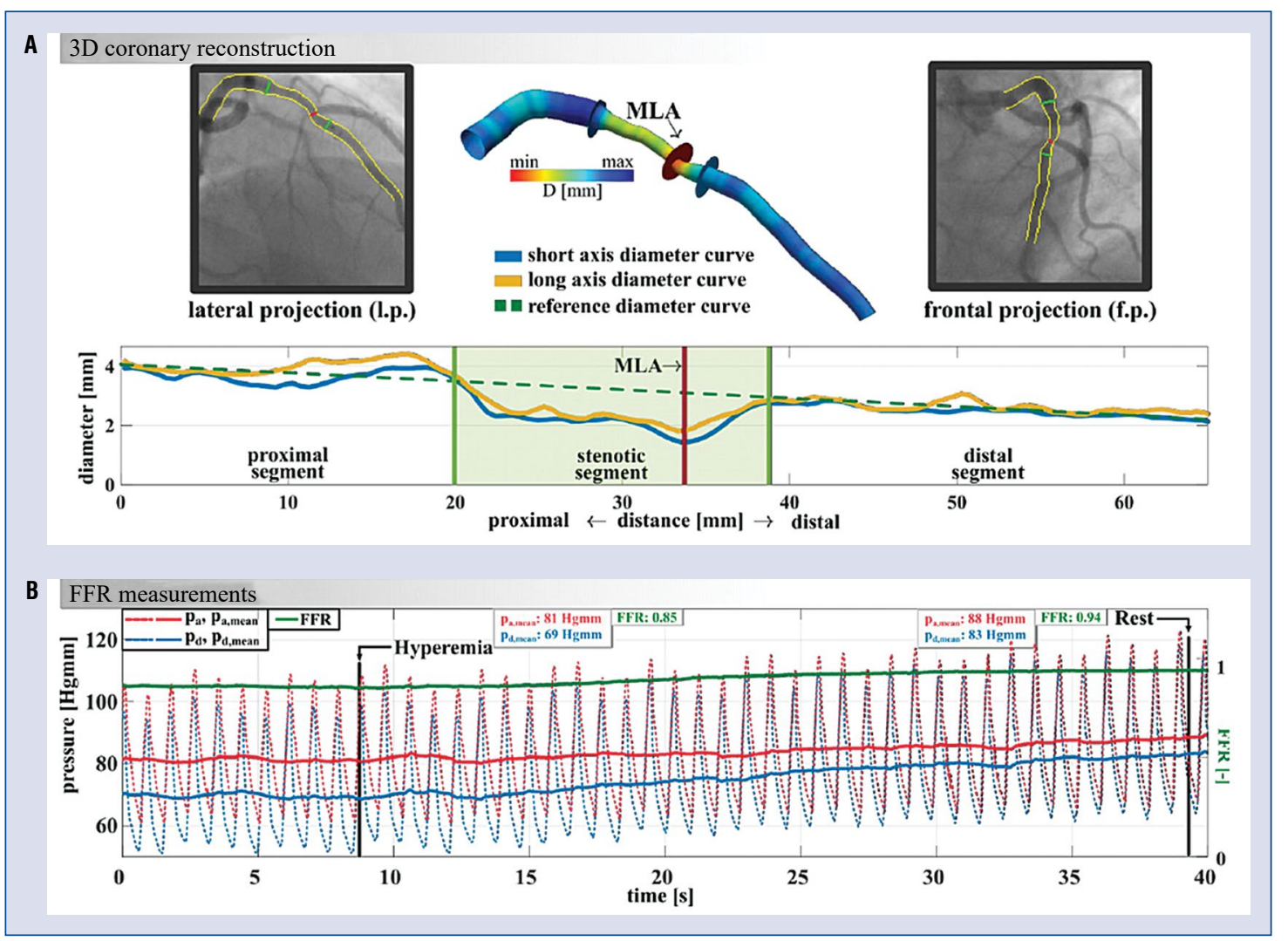

Figure 1. A. Detection of the segments by QAngio XA 3D. The stenotic segment lies between the two green lines, marked by shaded green color; B. Sample pressure trace after intracoronary adenosine injection. Red line: proximal (aortic) instantaneous pressure trace, red dashed line: moving average of the proximal pressure, blue line: instantaneous pressure trace distally to the coronary stenosis, blue dashed line: moving average of the distal pressure, green line: the ratio of the average distal and the proximal pressure (FFR); min — minimum; max — maximum; 3D — three-dimensional; D — diameter; $\mathrm{mm}$ - millimeter; MLA — minimal lumen area; Hgmm — millimeter of mercury; $\mathrm{p}_{\mathrm{a} \text { mean }}-$ mean pressure value in the proximal lumen area; $\mathrm{p}_{\mathrm{d} \text { mean }}-$ mean pressure value in the distal lumen area; FFR - fractional flow reserve; s - second.

Health Authorisation and Administrative Procedures (Project identification number: 44270/2013/ OTIG). Patient characteristics can be found in Table 1.

\section{Fractional flow reserve measurement}

Fractional flow reserve measurements were performed using a pressure-sensor guidewire via a 6 French $(\mathrm{F})$ guiding catheter. First, the target artery was passed with an intracoronary pressure wire (RADI Medical, Uppsala, Sweden). After calibrating the wire, pressures were equalized with the sensor positioned at the level of the catheter tip. The sensor was then advanced through the coronary stenosis and measurements were performed approximately $2 \mathrm{~cm}$ distal to the lesion. 150-200 $\mu \mathrm{g}$ intracoronary adenosine was administered, and the ratio of the distal coronary pressure $\left(p_{d}\right)$ and the aortic pressure $\left(p_{a}\right)$ gave the FFR (Fig. 1B, left-hand side, green line). The resting pressure ratio was measured after the disappearance of the adenosine effect (defined by returning to the same pressure trace as before the adenosine injection) (Fig. 1B, right-hand side).

\section{Three-dimensional quantitative coronary reconstruction}

The invasive coronary angiography was followed by offline 3D angiographic reconstruction using validated software (e.g., QAngio XA Research Edition 1.0, Medis Specials bv, Leiden) [20-23]. During the automatic reconstructions, two angiographic recordings of good quality were used with at least $25^{\circ}$ difference in angle. A target vessel segment in $3 \mathrm{D}$, starting from the coronary orifice to the location of the pressure wire sensor, was generated for further analysis. Subsequent to the $3 \mathrm{D}$ reconstruction, parameters required for the 
Table 1. Patient characteristics.

\begin{tabular}{|c|c|}
\hline Characteristics, Unit & Data \\
\hline \multicolumn{2}{|l|}{ General } \\
\hline Number of patients, & 9 \\
\hline Female, $\mathrm{n}$ & 5 \\
\hline Mean age (SD), years (years) & $68.8(8.2)$ \\
\hline \multicolumn{2}{|l|}{ CAD risk factors } \\
\hline Hypertonia, n (\%) & $9(100)$ \\
\hline Diabetes mellitus, n (\%) & $5(55)$ \\
\hline Hyperlipidemia, n (\%) & $2(22)$ \\
\hline Chronic renal failure, $\mathrm{n}(\%)$ & $2(22)$ \\
\hline \multicolumn{2}{|l|}{ Cardiovascular conditions } \\
\hline Aortic valve stenosis, $\mathrm{n}(\%)$ & $3(33)$ \\
\hline Previous ACS, n (\%) & $2(22)$ \\
\hline \multicolumn{2}{|l|}{ Interrogated coronary artery } \\
\hline LAD, n (\%) & $6(66)$ \\
\hline LCx, n (\%) & $1(11)$ \\
\hline RCA, n (\%) & $3(33)$ \\
\hline \multicolumn{2}{|l|}{ FFR measurement } \\
\hline FFR (SD), mean value (mean value) & $0.76(0.14)$ \\
\hline
\end{tabular}

computations, including the proximal reference cross-sectional area, the average stenotic area, the distal reference area, the length of the proximal reference area, the stenotic area, the distal reference area, as well as the minimal lumen area were calculated using the aforementioned software. The average cross-sections are determined automatically by the angiographic software based on volume equivalence; i.e., the cross-sections are computed by dividing the segment volume by its length.

The software evaluates the short-axis diameter (Fig. 1A, blue curve) and the reference diameter curve (Fig. 1A, dashed green curve) to find the above measurements. The short-axis diameter curve is based on the ellipses along the centerline that defines the entire $3 \mathrm{D}$ reconstruction. The reference diameter curve is a linear approximation for a hypothetical healthy vessel. At first, the algorithm finds the minimal lumen area (MLA), marked with a red vertical line, this is the smallest cross-sectional area in the stenosed segment. Then the software finds the intersection between the short-axis diameter curve (blue) and the reference diameter curve (dashed green), both proximally and distally from the position of the MLA. These proximal and distal positions (marked with green vertical lines) are the boundaries of the stenosed segment. The
$A_{p}$ (proximal), $A_{s}$ (stenosis), and $A_{d}$ (distal) cross-sectional areas are calculated accordingly.

\section{Description of the simple method for the} calculation of $\mathrm{CFR}_{\text {Model }}\left(\mathrm{CFR}_{\mathrm{M}}\right)$

A flow resistance caused by an obstacle in a flow manifests itself in a pressure drop. As discussed above, the pressure drop, in general, has a linear term (as a function of the velocity or the flow rate), representing viscous friction losses $(f Q)$, and a quadratic term which represents separation losses $\left(s Q^{2}\right)$. The method called the "simple model" used here is the reformulation of the similar methods in [14, 15, 24, 25] (Fig. 2):

$$
\begin{gathered}
\Delta \rho_{\mathrm{t}}=\Delta \rho_{\text {visc } \text { prox }}+\Delta \rho_{\text {vis } s_{\text {sten }}}+\Delta \rho_{\text {sep }}+\Delta \rho_{\text {visc }} \text { dist } \\
=f_{\text {prox }} Q+f_{\text {sten }} Q+\mathrm{sQ}^{2}+f_{\text {dist }} Q
\end{gathered}
$$

$\mathrm{Q}$ is the volumetric flow rate; $\Delta p_{t}$ is the total pressure drop; $\Delta p_{v i s c(i)}$ are the friction-related pressure losses, respectively for the proximal, stenotic, and distal part; and $\Delta p_{s e p}$ is the separation-related pressure loss term of the idealized stenosis. Thus, $f_{i}$ is the linear coefficient in the viscous pressure loss terms, and $\mathrm{s}$ is the quadratic coefficient in the separation-related pressure loss term. A simple model, shown in Figure 2, consists of three segments: the proximal, the stenotic, and the distal segment. All three parts are straight, cylindrical, rigid-walled tube sections. The length of the stenotic segment, denoted by $L_{s}$ in the model, is defined in Figure 1 as the segment where the red real diameter curve deviates from the blue reference diameter curve at more than a certain threshold. The steps of the volumetric flow determination are described in the patent of the method: https://patents.google.com/ patent/WO2019175612A2/en.

\section{Computational fluid dynamics calculation for CFR}

Computational fluid dynamics (CFD) techniques to study coronary artery disease is a widely used and quickly advancing research tool [26] used in pre- [27-30] and post-stent-treatment analysis [31]. After 3D reconstruction by QAngio XA, the geometries were imported to MeshLab [20] for further pre-processing to have a smooth surface for the CFD analysis. The software package ANSYS 18.2. (ANSYS Inc. Canonsburg, USA) was used for the $3 \mathrm{D}$ CFD analysis $[21,22]$ using the CFX numerical solver.

The measured aorta and distal pressure profiles were imposed on the inlet and outlet in unsteady simulations, respectively. $C F R_{\text {unsteady }}\left(C F R_{U}\right)$ was 


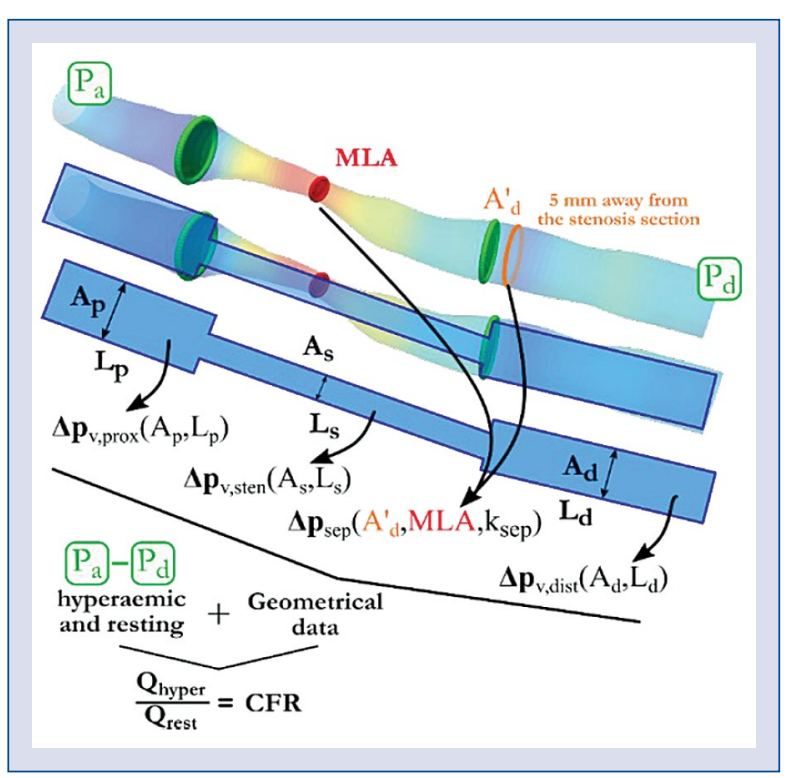

Figure 2. The simplified model of the target vessel is based on the three-dimensional quantitative coronary angiography geometry; CFR - coronary flow reserve; $\mathrm{MLA}$ - minimal lumen area; $\mathrm{A}_{\mathrm{d}}{ }$ - poststenotic vessel area-averaged on the segment $5 \mathrm{~mm}$ away from the stenotic segment; $A_{p}$ - proximal crosssectional area; $L_{p}$ - length of the proximal segment; $A_{s}$ - cross-sectional area of the stenosed segment; $L_{s}$ - length of the stenosis; $A_{d}$ - cross-sectional area of the distal segment; $L_{d}$ - length of the distal segment; $P_{a}-$ aortic pressure; $P_{d}-$ pressure distal to the stenosis; $\mathrm{Q}_{\text {hyper }}$ - flow rate under hyperemia; $\mathrm{Q}_{\text {rest }}$ - flow rate at rest; $\Delta \mathrm{p}_{\mathrm{v}, \text { prox }}$ - friction-related pressure loss in the proximal segment; $\Delta p_{v, s t e n}$ - friction-related pressure loss in the stenosis; $\Delta p_{v, d i s t}$ - friction-related pressure loss distal to the stenosis; $\Delta \mathrm{p}_{\mathrm{v}, \text { sep }}$ - separation-related pressure loss; $k_{\text {sep }}$ - correction for the entrance effects detailed in equation (6) [19].

calculated as the ratio of flow rates computed from resting and hyperemic conditions. The unsteady flow results were averaged within one heart cycle, and the $\mathrm{CFR}_{\text {steady }}\left(C F R_{S}\right)$ was calculated from these data.

Using the ANSYS software, all numerical meshes consisted of about 1.5 to 2 million numerical cells consisting mostly of linear tetrahedral cells and 5 prismatic layers adjacent to the wall for better near-wall resolution (see the inset image in Fig. 3). In the present simulations, the blood was assumed to be an incompressible Newtonian fluid, and the vessel walls to be rigid. The density and viscosity were set to be $1055 \mathrm{~kg} / \mathrm{m}^{3}$ and $3.5 \mathrm{mPas}$, respectively.

The setup of the steady-state simulations was as follows: the spatial discretization scheme was set to high resolution, which uses a blending function between first-order and second-order dis-

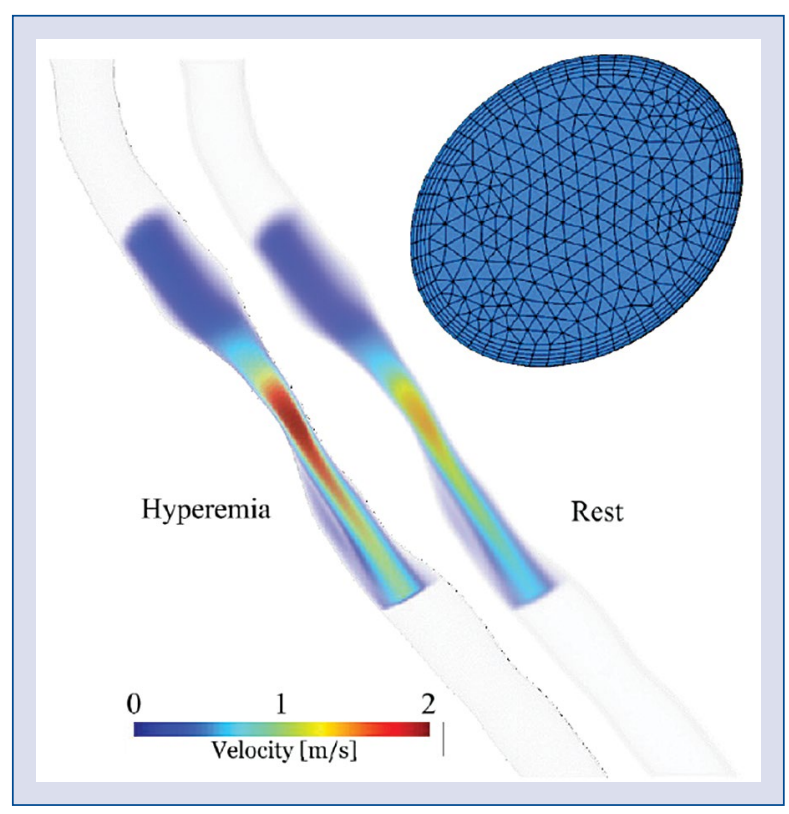

Figure 3. Sample display of the velocity field near the stenosis in the hyperemic and resting condition at the time instant of the highest velocity (case no. 5). The inset figure shows a sample cross-section of the mesh in the stenotic segment.

cretization according to the computation demands. The criterion $10^{-6}$ was set for the residuals, and the flow rate was monitored for convergence. A cycleaveraged pressure was calculated in the aorta and at the distal point in the coronary artery after the stenosis from the invasive pressure measurements. Since the flow rate is independent of the absolute pressure level, and depends only on the pressure difference, the difference of the two-cycle-averaged pressures were imposed as total pressure at the inlet to drive the flow within the vessel section, and zero $p_{a}$ static pressure at the outlet. To obtain the $\Delta p(Q)$ relationship and eventually the $\mathrm{f}$ and s coefficients, a set of 5 steady-state simulations were carried out with increasing inlet pressure values, including those corresponding to the resting and hyperemic conditions. A parabolic curve was fitted on these $5(\Delta p, Q)$ points, and the coefficients $\mathrm{f}$ and $\mathrm{s}$ were determined from the curve fitting. The measured aorta and distal pressure profiles were imposed on the inlet and outlet in the unsteady simulations, respectively. $C F R_{\text {unsteady }}\left(C F R_{U}\right)$ was calculated as the ratio of flow rates computed from the resting and hyperemic conditions.

Because the pressure detection in the aorta using a fluid-filled system, and in the coronary artery, is based on electrical conduction, the time signal of the pressure wire is shifted in time (comes 
Table 2. Calculated coronary flow reserve (CFR) values with the all three methods. $C F R_{M} / F F R$ was calculated based on [16].

\begin{tabular}{|c|c|c|c|c|}
\hline Case & $C F R_{M}$ & $C F R_{S}$ & $\mathrm{CFR}_{U}$ & $C F R_{M} / F F R$ \\
\hline 1 & 1.14 & 1.14 & 1.15 & 2.59 \\
\hline $2^{*}$ & 1.61 & 1.52 & 1.46 & 1.89 \\
\hline $3^{* *}$ & 1.27 & 1.32 & 1.02 & 1.74 \\
\hline $4^{* *}$ & 1.31 & 1.30 & 1.34 & 1.58 \\
\hline $5^{*}$ & 1.33 & 1.35 & 1.42 & 1.92 \\
\hline $6 * *$ & 1.08 & 1.08 & 1.06 & 1.46 \\
\hline 7 & 1.84 & 1.74 & 1.61 & 2.16 \\
\hline 8 & 2.67 & 2.70 & 3.27 & 3.22 \\
\hline 9 & 2.41 & 2.11 & 1.77 & 2.73 \\
\hline Average (SD) & $1.63(0.57)$ & $1.58(0.52)$ & $1.57(0.68)$ & $2.14(0.59)$ \\
\hline Correlation analysis: & & & $\mathbf{r}$ & $\mathbf{p}$ \\
\hline$C F R_{M}-C F R_{S}$ & & & 0.984 & $<10^{-5}$ \\
\hline$C F R_{M}-C F R_{U}$ & & & 0.876 & $<10^{-2}$ \\
\hline$C F R_{S}-C F R_{U}$ & & & 0.940 & $<10^{-3}$ \\
\hline
\end{tabular}

*Patient with previous acute coronary syndrome, FFR was measured in the culprit vessel; **Patients with severe aortic valve stenosis; FFR - fractional flow reserve; SD - standard deviation; CFR $_{S}$ - coronary flow reserve with steady calculation by ANSYS; CFR $\mathrm{R}_{U}$ - coronary flow reserve with unsteady calculation by ANSYS; CFR ${ }_{M}$ - coronary flow reserve calculated with simple model; $r-$ Pearson correlation coefficient

earlier) relative to the aortic pressure trace. This $2-5 \mathrm{~ms}$ shift is corrected before being used in the CFD simulations. A second-order backward Euler method was chosen for the temporal discretization scheme with adaptive time stepping to fulfill the CFL criterion of 1.0. Three cycles were simulated, and only the last cycle was post-processed to exclude any form of the initial transient. The unsteady flow results were then averaged within that one last heart cycle, and the $C F R_{\text {steady }}\left(C F R_{S}\right)$ was calculated from these data.

\section{Results}

In Table 2 a summary of the computed CFR values is reported.

The correlation between the CFR was determined by the simple equations and from the steady flow simulation $\left(C F R_{M}-C F R_{S}\right)$ was excellent $\left(\mathrm{r}=0.984, \mathrm{p}<10^{-5}\right)$. Table 2 also shows that the correlation between the results of the steady and the cycle-averaged unsteady simulations $\left(C F R_{S}-C F R_{U}\right)$ remained good and significant, with a Pearson correlation coefficient of 0.94 and $(\mathrm{p}<0.001)$. Finally, the correlation between the model values and unsteady simulations $\left(C F R_{M}\right.$ $\left.-C F R_{U}\right)$ remained pleasant $\left(\mathrm{r}=0.876, \mathrm{p}<10^{-2}\right)$.

Figure 4 shows the scatter plots of the comparisons and their corresponding Bland-Altman analysis for the simple method, steady and unsteady state simulations. The Bland-Altman analysis of the above-demonstrated methods did not systematically skew the values in the investigated range.

The $C F R_{M} / F F R$ was derived as an analogue to the microvascular reserve characterizing the microvascular state [16]. Values below 2.0 indicate a microvascular dysfunction. All 5 of those patients with $C F R_{M} / F F R<2$ had clinical evidence for microvascular disease (Table 2 ).

The overall pressure loss $\left(\Delta p_{t}\right.$, the sum effect of the friction- $(f Q)$ and separation- $\left(s Q^{2}\right)$ related pressure loss types) of each case, derived by the simple model, is smaller than that calculated by CFD simulation. In Figure 5, flow rate as a function of the pressure-drop $(Q(\Delta p))$, the diagram is shown containing 3 cases with decreasing resistances. The solid and dashed curves are defined by the simple model and the CFD simulations, respectively. The lowest resistance (lowest $\mathrm{f}$ and s coefficients) was obtained for Case 8 by the simple model (the solid blue steep curve in Fig. 5), rendering the flow rate calculation to be quite sensitive.

While the actual numerical values of the quadratic losses are rather underestimated by the simple model because of the fact that vessel tortuosity and lesion eccentricity could be the sources for separation-related losses but these are not incorporated into the simple model yet, the 


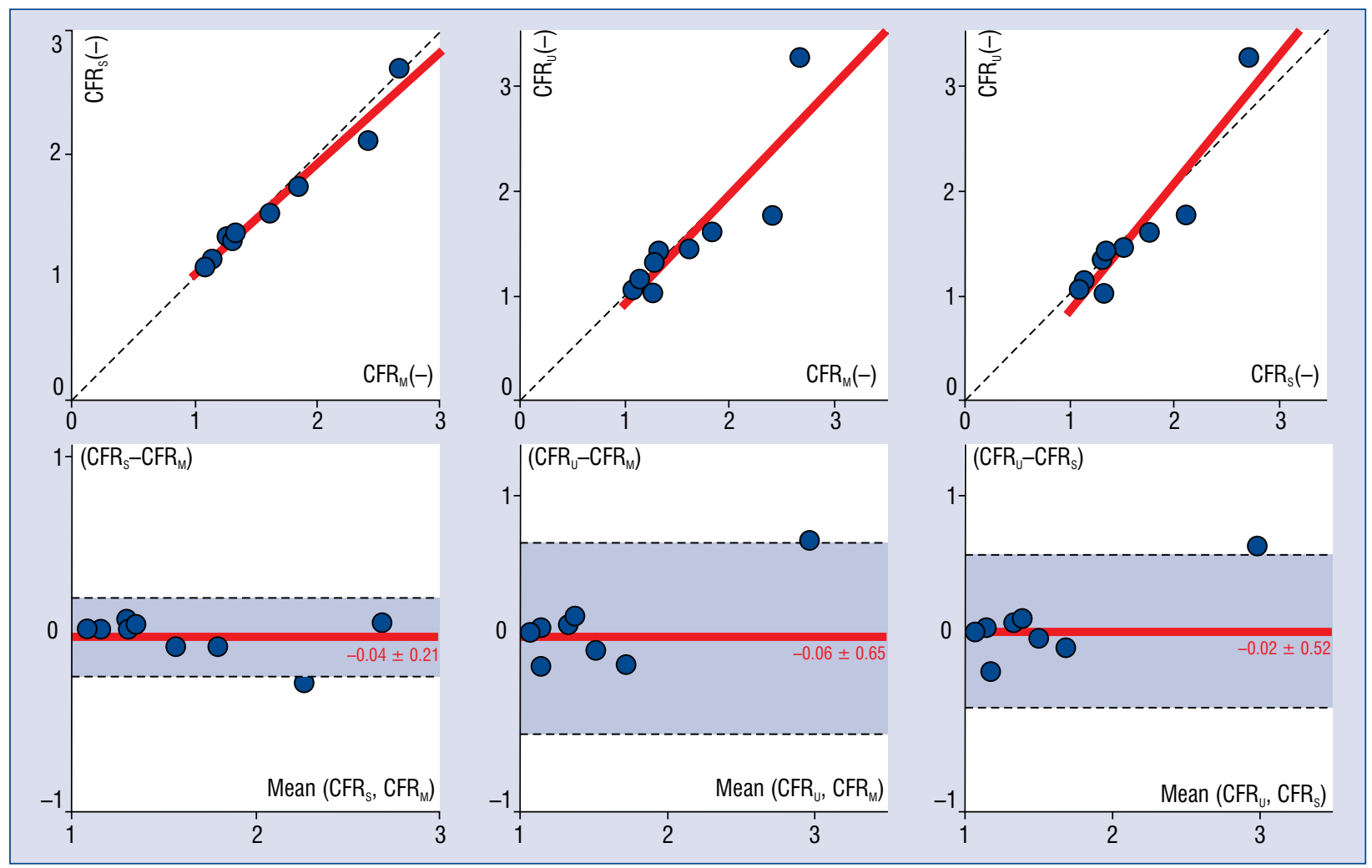

Figure 4. Correlations between the models and their corresponding Bland-Altman plots. Correlation coefficients can be found on the bottom of Table 2; CFR $_{S}$ - coronary flow reserve with steady calculation by ANSYS; CFR - coro- $^{-}$ nary flow reserve with unsteady calculation by ANSYS; CFR $\mathrm{M}_{\mathrm{M}}$ - coronary flow reserve calculated with the simple model.

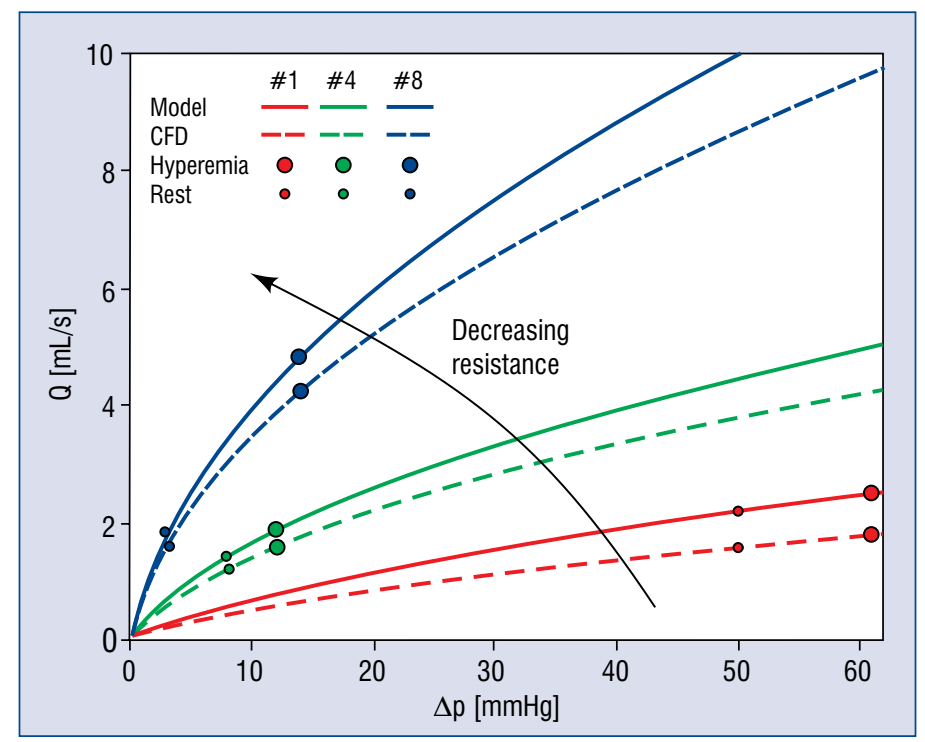

Figure 5. Flow rate as a function of the pressure difference $Q(\Delta p)$. Three distinct cases that represent the lowest (blue), middle (green) and largest (red) resistances in the studied population. The solid and dashed line depicts the curve defined by the simple model and steady CFD simulations by ANSYS, respectively. The marked points on each curve are the solutions of the flow equations by substituting the pressure drop of the given case from the resting and hyperemic condition; CFD — computational fluid dynamics calculation by ANSYS; $\mathrm{Q}$ - flow rate; $\mathrm{mL} / \mathrm{s}$ - milliliter/ /second; CFR - coronary flow reserve; $\Delta \mathrm{p}$ - pressure loss; $\mathrm{mmHg}$ - millimeter of mercury. 
difference between the CFR values is small, being that the CFR is a ratio-type variable.

The present results for unsteady and steady flow simulations are in line with earlierstudies [23, $32]$, that also relied on using pressure histories.

\section{Discussion}

Morris et al. [33] recently published a method for absolute flow calculation using similar pressure input data for fluid dynamic computation by ANSYS software (QCFD), this is the simplified method used herein. However, our simple method only requires an Excel sheet for the flow calculations, and - according to available research, the first one of its kind - furthermore, it is, to a large extent, automated and requires only a couple of minutes to perform the whole procedure.

The full 3D CFD simulations using the programs mentioned above are computationally demanding and need professional pre-processing. The pre-processing and meshing take around half an hour. In contrast, the set-up and the computation of the steady-state and unsteady analysis takes around 1 hour and 2 days, respectively, utilizing a 4-core 16 GB RAM computer. This time span makes it unrealistic to use this workflow outside the framework of clinical research. On the other hand, the proposed method with a simplified calculation of the CFR requires the most detailed possible data and shows results comparable to that of detailed CFD modeling. Therefore, this simple calculation method seems suitable for clinical applications that offer a more comprehensive evaluation than FFR measurement alone.

Apart from its small sample size and retrospective nature, some further limitations of the present study should be mentioned. First, the blood is a suspension of particles and the blood plasma, and on a microscopic level, it behaves like a non-Newtonian fluid; however, in the present CFD simulations, a Newtonian approach was used. This approximation is widely accepted and used by the hemodynamic research community.

The CFD simulations used "single tube" 3D reconstructions discarding side branches of the main segments. In this way, the same volumetric flow rate in the reconstructed coronary artery was assumed, not considering flow losses through side branches. Others showed that excluding side branches significantly affects calculating flow rates but can still be used to calculate a ratio-type value [34].

\section{Conclusions}

It was demonstrated herein, that during a routine FFR investigation, the measured intracoronary pressure data and $3 \mathrm{D}$ reconstructed coronary angiography offer a feasible means of calculating the volumetric flow rate and the CFR. The flow calculation needs very little time and can provide online comprehensive flow-pressure relation. Validation of the current simple calculation technique has been carried out for coronary lesions of a small-sized patient population in a widely accepted finite volume solver ANSYS CFX.

The CFR and FFR (CFR/FFR) relation will be an indicator of microvascular function analog to microvascular resistance reserve. In the current study, some patients showed markedly low CFR values. Among them, most had stenotic aortic valves. This observation is in line with the earlier data showing that CFR values in patients with severe aorta stenosis are radically reduced despite visually intermediate coronary lesions [35, 36].

According to these values, the clinical decision-making on medical therapy or percutaneous/ /surgical revascularization could be guided more precisely. The CFR results from simplified calculations show strong agreement with those from steady flow simulations and thus may be accurate enough for clinical applications. For a comparison of the results a larger size study with established clinical flow investigation, e.g., by intracoronary Doppler wire measurements, is planned.

\section{Acknowledgments}

The research reported in this paper was supported by the BME NC TKP2020 grant of NKFIH Hungary. Áron Üveges was supported by the ÚNKP-20-03 New National Excellence, Program of the Ministry for Innovation and Technology from the source of the National Research, Development, and Innovation Fund.

Conflict of interest: The patent of the method described in this paper was issued by the Hungarian Intellectual Property Office (Appl. No.: PCT/ /HU2019/050008, applicant: University of Debrecen). Zsolt Köszegi is the inventor of this patent. The other authors reported no conflict of interest.

\section{References}

1. Pijls NHJ, van Schaardenburgh P, Manoharan G, et al. Percutaneous coronary intervention of functionally nonsignificant stenosis: 5-year follow-up of the DEFER Study. J Am Coll Cardiol. 2007; 
49(21): 2105-2111, doi: 10.1016/j.jacc.2007.01.087, indexed in Pubmed: 17531660.

2. Tonino PAL, Patricio L, De Bruyne B, et al. Fractional flow reserve versus angiography for guiding percutaneous coronary intervention. N Engl J Med. 2009; 28: 229-230.

3. Johnson NP, Tóth GG, Lai D, et al. Prognostic value of fractional flow reserve: linking physiologic severity to clinical outcomes. J Am Coll Cardiol. 2014; 64(16): 1641-1654, doi: 10.1016/j. jacc.2014.07.973, indexed in Pubmed: 25323250.

4. Neumann FJ, Sousa-Uva M, Ahlsson A, et al. 2018 ESC/EACTS Guidelines on myocardial revascularization. Eur Heart J. 2019; 40: 87-165, doi: 10.1093/eurheartj/ehy394, indexed in Pubmed: 30165437 .

5. De Bruyne B, Fearon WF, Pijls NHJ, et al. Fractional flow reserve-guided PCI for stable coronary artery disease. N Engl J Med. 2014; 371(13): 1208-1217, doi: 10.1056/NEJMoa1408758, indexed in Pubmed: 25176289.

6. Gan LM, Wikström J, Fritsche-Danielson R. Coronary flow reserve from mouse to man--from mechanistic understanding to future interventions. J Cardiovasc Transl Res. 2013; 6(5): 715-728, doi: 10.1007/s12265-013-9497-5, indexed in Pubmed: 23877202.

7. Komócsi A, Pintér T, Faludi R, et al. Overlap of coronary disease and pulmonary arterial hypertension in systemic sclerosis. Ann Rheum Dis. 2010; 69(1): 202-205, doi: 10.1136/ard.2008.096255, indexed in Pubmed: 19158116.

8. Johnson NP, Kirkeeide RL, Gould KL. Is discordance of coronary flow reserve and fractional flow reserve due to methodology or clinically relevant coronary pathophysiology? JACC Cardiovasc Imaging. 2012; 5(2): 193-202, doi: 10.1016/j.jcmg.2011.09.020, indexed in Pubmed: 22340827.

9. Barbato E, Aarnoudse W, Aengevaeren WR, et al. Validation of coronary flow reserve measurements by thermodilution in clinical practice. Eur Heart J. 2004; 25(3): 219-223, doi: 10.1016/j. ehj.2003.11.009, indexed in Pubmed: 14972422.

10. Everaars H, de Waard GA, Driessen RS, et al. Doppler Flow Velocity and Thermodilution to Assess Coronary Flow Reserve: A Head-to-Head Comparison With [O]HO PET. JACC Cardiovasc Interv. 2018; 11(20): 2044-2054, doi: 10.1016/j.jcin.2018.07.011, indexed in Pubmed: 30268877.

11. Williams RP, de Waard GA, De Silva K, et al. Doppler versus thermodilution-derived coronary microvascular resistance to predict coronary microvascular dysfunction in patients with acute myocardial infarction or stable angina pectoris. Am J Cardiol. 2018; 121(1): 1-8, doi: 10.1016/j.amjcard.2017.09.012, indexed in Pubmed: 29132649.

12. Cortigiani L, Rigo F, Gherardi S, et al. Coronary flow reserve during dipyridamole stress echocardiography predicts mortality. JACC Cardiovasc Imaging. 2012; 5(11): 1079-1085, doi: 10.1016/j.jcmg.2012.08.007, indexed in Pubmed: 23153906.

13. Murthy VL, Naya M, Foster CR, et al. Improved cardiac risk assessment with noninvasive measures of coronary flow reserve. Circulation. 2011; 124(20): 2215-2224, doi: 10.1161/CIRCULATIONAHA.111.050427, indexed in Pubmed: 22007073.

14. Di Marlo C, Gil R, Feyter Pde, et al. Utilization of translesional hemodynamics: Comparison of pressure and flow methods in stenosis assessment in patients with coronary artery disease. Catheter Cardiovasc Diagn. 1996; 38(2): 189-201, doi: 10.1002/ (sici)1097-0304(199606)38:2<189::aid-ccd17>3.0.co;2-e.

15. Gould KL, Johnson NP, Bateman TM, et al. Anatomic versus physiologic assessment of coronary artery disease: Role of coronary flow reserve, fractional flow reserve, and positron emission tomography imaging in revascularization decision-making. J Am Coll Cardiol. 2013; 62: 1639-1653.

16. Garcia D, Harbaoui B, van de Hoef TP, et al. Relationship between FFR, CFR and coronary microvascular resistance - Practical implications for FFR-guided percutaneous coronary intervention. PLoS One. 2019; 14(1): e0208612, doi: 10.1371/journal. pone.0208612, indexed in Pubmed: 30616240.

17. Martini R. The compelling arguments for the need of microvascular investigation in COVID-19 critical patients. Clin Hemorheol Microcirc. 2020; 75(1): 27-34, doi: 10.3233/CH-200895, indexed in Pubmed: 32568186.

18. Nägele MP, Haubner B, Tanner FC, et al. Endothelial dysfunction in COVID-19: Current findings and therapeutic implications. Atherosclerosis. 2020; 314: 58-62, doi: 10.1016/j.atherosclerosis.2020.10.014, indexed in Pubmed: 33161318.

19. Szabó GT, Üveges Á, Tar B, et al. The holistic coronary physiology display: calculation of the flow separation index in vessel-specific individual flow range during fractional flow reserve measurement using 3D coronary reconstruction. J Clin Med. 2021; 10(9), doi: 10.3390/jcm10091910, indexed in Pubmed: 33924961.

20. Cignoni P, Callieri M, Corsini M, Dellepiane M, Ganovelli F, Ranzuglia G. MeshLab: An open-source mesh processing tool. In Proceedings of the 6th Eurographics Italian Chapter Conference 2008 - Proceedings; 2008; 129-136.

21. ANSYS Inc. ANSYS CFX 18.22018.

22. Závodszky G, Paál G. Validation of a lattice Boltzmann method implementation for a 3D transient fluid flow in an intracranial aneurysm geometry. Int J Heat Fluid Flow. 2013; 44: 276-283, doi: 10.1016/j.ijheatfluidflow.2013.06.008.

23. Morris PD, Silva Soto DA, Feher JFA, et al. Fast virtual fractional flow reserve based upon steady-state computational fluid dynamics analysis: results from the virtu-fast study. JACC Basic Transl Sci. 2017; 2(4): 434-446, doi: 10.1016/j.jacbts.2017.04.003, indexed in Pubmed: 28920099.

24. Young D, Tsai F. Flow characteristics in models of arterial stenosis II. Unsteady Flow. 1973; 6(4): 547-559.

25. Tar B, Jenei C, Dezsi CA, et al. Less invasive fractional flow reserve measurement from 3-dimensional quantitative coronary angiography and classic fluid dynamic equations. EuroIntervention. 2018; 14(8): 942-950, doi: 10.4244/EIJ-D-17-00859, indexed in Pubmed: 29488883.

26. Gray RA, Pathmanathan P. Patient-Specific cardiovascular computational modeling: diversity of personalization and challenges. J Cardiovasc Transl Res. 2018; 11(2): 80-88, doi: 10.1007/ s12265-018-9792-2, indexed in Pubmed: 29512059.

27. Papafaklis MI, Muramatsu T, Ishibashi Y, et al. Fast virtual functional assessment of intermediate coronary lesions using routine angiographic data and blood flow simulation in humans: comparison with pressure wire - fractional flow reserve. EuroIntervention. 2014; 10(5): 574-583, doi: 10.4244/EIJY14M07_01, indexed in Pubmed: 24988003.

28. Tu S, Barbato E, Köszegi Z, et al. Fractional flow reserve calculation from 3-dimensional quantitative coronary angiography and TIMI frame count: a fast computer model to quantify the functional significance of moderately obstructed coronary arteries. JACC Cardiovasc Interv. 2014; 7(7): 768-777, doi: 10.1016/j. jcin.2014.03.004, indexed in Pubmed: 25060020.

29. Zarins CK, Taylor CA, Min JK. Computed fractional flow reserve (FFTCT) derived from coronary CT angiography. J Cardiovasc Transl Res. 2013; 6(5): 708-714, doi: 10.1007/s12265-013-9498-4, indexed in Pubmed: 23934536. 


\section{Cardiology Journal}

30. Liu G, Wu J, Ghista DN, et al. Hemodynamic characterization of transient blood flow in right coronary arteries with varying curvature and side-branch bifurcation angles. Comput Biol Med. 2015; 64: 117-126, doi: 10.1016/j.compbiomed.2015.06.009, indexed in Pubmed: 26164032.

31. Chiastra C, Migliori S, Burzotta F, et al. Patient-Specific modeling of stented coronary arteries reconstructed from optical coherence tomography: towards a widespread clinical use of fluid dynamics analyses. J Cardiovasc Transl Res. 2018; 11(2): 156-172, doi: 10.1007/s12265-017-9777-6, indexed in Pubmed: 29282628.

32. Fossan FE, Sturdy J, Müller LO, et al. Uncertainty quantification and sensitivity analysis for computational FFR estimation in stable coronary artery disease. Cardiovasc Eng Technol. 2018; 9(4): 597-622, doi: 10.1007/s13239-018-00388-w, indexed in Pubmed: 30382522.

33. Morris PD, Gosling R, Zwierzak I, et al. A novel method for measuring absolute coronary blood flow and microvascular re- sistance in patients with ischaemic heart disease. Cardiovasc Res. 2021; 117(6): 1567-1577, doi: 10.1093/cvr/cvaa220, indexed in Pubmed: 32666101.

34. Gosling RC, Sturdy J, Morris PD, et al. Effect of side branch flow upon physiological indices in coronary artery disease. J Biomech. 2020; 103: 109698, doi: 10.1016/j.jbiomech.2020.109698, indexed in Pubmed: 32151377.

35. Garcia D, Camici PG, Durand LG, et al. Impairment of coronary flow reserve in aortic stenosis. J Appl Physiol (1985). 2009; 106(1): 113-121, doi: 10.1152/japplphysiol.00049.2008, indexed in Pubmed: 18974370.

36. Gutiérrez-Barrios A, Gamaza-Chulián S, Agarrado-Luna A, et al. Invasive assessment of coronary flow reserve impairment in severe aortic stenosis and ecochadiographic correlations. Int J Cardiol. 2017; 236: 370-374, doi: 10.1016/j.ijcard.2017.01.150, indexed in Pubmed: 28169057. 